\title{
Analysis of Laser Pulse Chirping in Mode-Locked Vertical-Cavity Surface-Emitting Lasers
}

\author{
Wenbin Jiang, Dennis J. Derickson, Member, IEEE, and John E. Bowers, Fellow, IEEE
}

\begin{abstract}
Mode-locked vertical-cavity lasers have a large cross-sectional area and consequently a large saturation energy and large peak powers. We analyze excess optical bandwidth generation in these lasers and find that self-phase modulation due to optical pumping and gain saturation is the dominant factor in inducing laser pulse chirping. The large magnitude of the chirp makes intracavity prism-pair compensation difficult. Adjustment of the cavity length has a major impact on the pulse chirping, as observed experimentally. Proper adjustment can result in a large linear frequency chirp which can be compensated using external pulse compression techniques.
\end{abstract}

\section{INTRODUCTION}

M ODE locking of vertical-cavity surface-emitting lasers (VCSEL) has been reported with both GaAs [1] and InGaAs [2], [3] semiconductor active materials using synchronous optical pumping. Because of the large active cross-sectional area in the VCSEL, pulses with the peak power over $100 \mathrm{~W}$ have been obtained. This peak power is about two orders of magnitude larger than that directly from mode-locked edge emitting semiconductor lasers. External cavity chirp compensation of mode-locked VCSEL's has been able to produce subpicosecond laser pulses [1]-[3]. The high peak power has also allowed the use of soliton compression techniques, in which the pulses have been shortened to 21 fs [4]. A new generation of mode-locked semiconductor lasers using vertical cavity structures arranged into 2-D array will be capable of generating short optical pulses with kilowatts of peak power.

It has been found, however, that the pulses generated in this type of lasers are usually highly chirped and have a large time-bandwidth product. This large chirping makes it difficult to directly generate subpicosecond pulses from the lasers. In this paper, we will discuss the origin and the magnitude of the laser pulse chirp. The analysis is applicable to both GaAs and InGaAs systems, but we will specifically analyze the GaAs laser as an example.

Fig. 1 shows the laser structure used for the modelocked GaAs VCSEL. The laser gain is provided by 120 $\mathrm{GaAs}-\mathrm{Al}_{0.3} \mathrm{Ga}_{0.7} \mathrm{As}$ quantum wells. The well width is 150 $\AA$ and the barrier width is $70 \AA$. The total effective length of the gain will thus be $1.8 \mu \mathrm{m}$. The center wavelength

Manuscript received May 7, 1992; revised October 19, 1992. This work was supported by DARPA and Rome Laboratories.

The authors are with the Department of Electrical and Computer Engineering, University of California, Santa Barbara, CA 93106

IEEE Log Number 9207846.

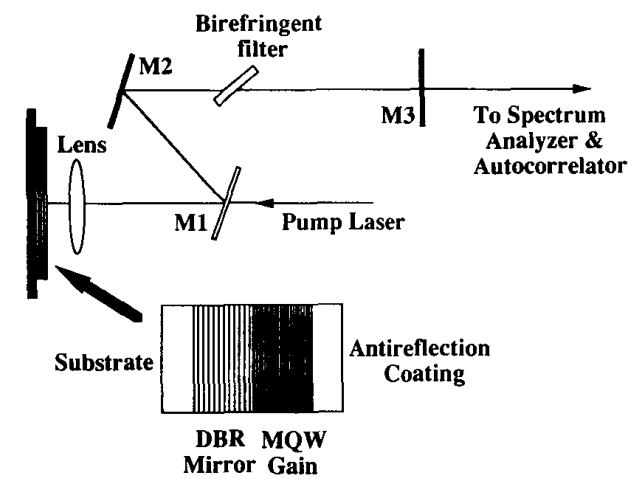

Fig. 1. Mode-locked vertical-cavity surface-emitting laser (VCSEL). $M 1$ is a dichroic mirror, $M 2$ is a high reflective mirror, and $M 3$ is an output coupler with reflectivity of $97 \%$. The focal length of the lens is $38.1 \mathrm{~mm}$.

of the gain is $860 \mathrm{~nm}$ at room temperature. One end mirror of the $Z$-shaped cavity is an epitaxially grown AlAs$\mathrm{Al}_{0.2} \mathrm{Ga}_{0.8}$ As distributed feedback mirror (DBR) on a GaAs substrate with the peak reflectivity over $99 \%$. The other end mirror is an external cavity dielectric mirror as the output coupler. The laser is synchronously pumped at 800 $\mathrm{nm}$ by a mode-locked dye laser. The repetition rate of the pump is $80 \mathrm{MHz}$ and the pump pulsewidth is $10 \mathrm{ps}$ with the average pump power up to $200 \mathrm{~mW}$ [1]. Within this mode-locked laser, the following mechanisms will affect the laser pulse chirp:

a) the self-phase modulation (SPM) caused by gain saturation and pulsed optical pumping

b) the phase dispersion caused by the finite gain shape

c) the dispersion caused by the DBR structure of both the semiconductor mirror and the dielectric mirrors

d) the material dispersion of the intracavity components.

The dispersion caused by the DBR structure has been well analyzed [5], [6] and is on the order of $10^{-28} \mathrm{~s}^{2}$. It varies depending on the structure and the growth. The material dispersion for the related semiconductor materials in the transparent region and other intracavity components like fused silica glass is also known [7], [8]. They will be on the order of $10^{-28} \mathrm{~s}^{2}$ for $2 \mu \mathrm{m}$ thick GaAs-AlGaAs [7] or for $5 \mathrm{~mm}$ thick fused silica [8]. In this paper, we mainly demonstrate the laser chirp caused by a) and b) after a single pass through the cavity. In Section II we will discuss the laser SPM caused by the gain saturation and the 
pulse pumping. In Section III we will discuss the phase dispersion caused by the finite gain shape. The laser pulse evolution in the laser cavity will be discussed in Section IV. We will compare the experimental results with the theoretical results in Section $\mathrm{V}$ and conclude the paper in Section VI.

\section{Self-Phase-Modulation of Laser Pulse}

In this section we will discuss the SPM caused by the gain saturation and the optical pulse pumping. Agrawal's treatment of pulse propagation in a semiconductor laser amplifier [9] has been modified to include an optical pulse pumping term. We start from the equation

$$
\begin{aligned}
& \frac{\partial A(z, t)}{\partial z}+\frac{1}{v_{g}} \frac{\partial A(z, t)}{\partial t}+\frac{i}{2} \beta_{2} \frac{\partial^{2} A(z, t)}{\partial t^{2}} \\
& =\frac{1}{2}(1-i \alpha) g(z, t) A(z, t)-\frac{1}{2} \alpha_{i n t} A(z, t)
\end{aligned}
$$

where $A(z, t)$ is the slowly varying pulse amplitude envelope of the electric field

$$
\mathbf{E}(z, t)=\mathbf{e}_{\mathbf{x}} \frac{A(z, t)}{\sqrt{\sigma}} \exp \left[i\left(k_{0} z-\omega_{0} t\right)\right]
$$

with $A(z, t)$ normalized so that $|A(z, t)|^{2}$ stands for the instantaneous laser power, $v_{g}$ is the group velocity, $\beta_{2}$ is the material dispersion parameter, $g(z, t)$ is the amplifier power gain, $\alpha_{i n t}$ is the internal scattering loss and $\alpha$ is the linewidth enhancement factor. In (2), $\mathbf{e}_{\mathbf{x}}$ is the polarization unit vector, $\sigma$ is the active cross-sectional area, $k_{0}$ is the free-space wave vector of the laser pulse, and $\omega_{0}$ is the optical central frequency. The gain is modeled to have a linear relationship to carrier density as shown by

$$
g(z, t)=a\left[N(z, t)-N_{0}\right]
$$

where $a$ is the differential gain coefficient and $N_{0}$ is the carrier density value at transparency. We have taken the transverse mode confinement factor $\Gamma$ as unity, which is reasonable for the VCSEL.

The carrier density variation with time at position $z$ in the gain medium is determined by the rate equation

$$
\begin{aligned}
\frac{\partial N(z, t)}{\partial t}= & \frac{\alpha_{p}(z, t)}{\hbar \omega_{p} \sigma} P_{p}(z, t+\Delta t)-\frac{N(z, t)}{\tau_{c}} \\
& -\frac{g(z, t)}{\hbar \omega_{0} \sigma}|A(z, t)|^{2}+D \frac{\partial^{2} N(z, t)}{\partial z^{2}}
\end{aligned}
$$

where $P_{p}(z, t+\Delta t)$ is the pump laser power, $\alpha_{p}(z, t)$ is the absorption of the active material to the pump pulse at pump frequency $\omega_{p}, \tau_{c}$ is the carrier lifetime, and $D$ is the carrier diffusion coefficient. $\Delta t$ determines the position of the pump pulse relative to the laser pulse. If $\Delta t>0$, the pump pulse arrives earlier than the laser pulse, and if $\Delta t$ $<0$, the laser pulse arrives earlier than the pump pulse. $\alpha_{p}(z, t)$ is assumed to be linearly proportional to the car- rier density $N(z, t)$, as shown by

$$
\alpha_{p}(z, t)=-b\left[N(z, t)-N_{p 0}\right]
$$

where $b$ is the differential gain coefficient at the pumping frequency $\omega_{p}$ and $N_{p 0}$ is the transparency carrier density value at $\omega_{p}$.

The $\beta_{2}$ term in (1) will be ignored and the phase dispersion effect on the chirp formation of the laser pulse due to the finite gain shape will be addressed in Section III. We assume $\alpha_{i n t} \ll g(z, t)$, and ignore this term in (1). The carrier density is assumed nearly uniform in the $z$ direction since the net active layer is only $1.8 \mu \mathrm{m}$ thick and is bleached by the high optical pumping of around $150 \mathrm{~mW}$ at the wavelength of $800 \mathrm{~nm}$, so the diffusion term in (4) is ignored. The term including $\tau_{c}$ in (4) can be ignored when the optical pulsewidths are much shorter than the carrier recombination lifetime. Under these conditions, (1) and (4) is simplified to

$$
\begin{aligned}
\frac{\partial A(z, \tau)}{\partial z}= & \frac{1}{2}(1-i \alpha) g(z, \tau) A(z, \tau) \\
\frac{\partial N(z, \tau)}{\partial \tau}= & \frac{\alpha_{p}(z, \tau)}{\hbar \omega_{p} \sigma} P_{p}(z, \tau+\Delta \tau) \\
& -\frac{g(z, \tau)}{\hbar \omega_{0} \sigma}|A(z, \tau)|^{2}
\end{aligned}
$$

where the local time $\tau=t-z / v_{g}$ has been introduced. Similar to the derivation of (6) for $A(z, \tau)$ from (1), we may obtain the equation for the pump $P_{p}(z, \tau+\Delta \tau)$ as shown by

$$
\frac{P_{p}(z, \tau+\Delta \tau)}{\partial z}=-\alpha_{p}(z, \tau) P_{p}(z, \tau+\Delta \tau) .
$$

With (3) in (7), we have

$$
\begin{aligned}
\frac{\partial g(z, \tau)}{\partial \tau}= & \frac{\alpha_{p}(z, \tau)}{\gamma E_{p \mathrm{sat}}} P_{p}(z, \tau+\Delta \tau) \\
& -\frac{g(z, \tau)}{E_{\mathrm{sat}}}|A(z, \tau)|^{2}
\end{aligned}
$$

where

$$
\begin{gathered}
E_{\mathrm{sat}}=\frac{\hbar \omega_{0} \sigma}{a} \\
E_{p \mathrm{sat}}=\frac{\hbar \omega_{p} \sigma}{b}
\end{gathered}
$$

are the saturation energies of the gain medium at laser frequency $\omega_{0}$ and pump frequency $\omega_{p}$, respectively, and

$$
\gamma=b / a
$$

is the ratio between the differential gain coefficients at $\omega_{p}$ and $\omega_{0}$. From (3) and (5), it is found that $\alpha_{p}(z, \tau)$ and $g(z$, $\tau)$ are related by

$$
\alpha_{p}(z, \tau)=-\gamma g(z, \tau)+b\left(N_{p 0}-N_{0}\right)
$$


Our discussions will be based on (6), (8), (9), and (13). Both the pump and the laser input pulses will be assumed to be Gaussian, $E_{0} /\left(\sqrt{\pi} \tau_{0}\right) \exp \left[-\left(\tau / \tau_{0}\right)^{2}\right]$, where $E_{0}$ is the pulse energy and $2 \sqrt{\ln 2} \tau_{0}$ is the FWHM (full width at half maximum) pulsewidth.

We rewrite the normalized complex electric field $A(z$, $\tau$ ) in terms of the real values $P(z, \tau)$, the laser power, and $\phi(z, \tau)$, the phase,

$$
A(z, \tau)=\sqrt{P(z, \tau)} \exp [i \phi(z, \tau)]
$$

so that (6) becomes

$$
\begin{aligned}
& \frac{\partial P(z, \tau)}{\partial z}=g(z, \tau) P(z, \tau) \\
& \frac{\partial \phi(z, \tau)}{\partial z}=-\frac{\alpha(z, \tau)}{2} g(z, \tau) .
\end{aligned}
$$

Equations (15) and (16) can be analytically solved, as shown by

$$
\begin{aligned}
& P_{\text {out }}(\tau)=P_{\text {in }}(\tau) e^{G(\tau)} \\
& \phi_{\text {out }}(\tau)=\phi_{\text {in }}(\tau)-\frac{1}{2} \alpha G(\tau)
\end{aligned}
$$

where $P_{i n}(\tau)$ and $P_{\text {out }}(\tau)$ stand for the power of the input and output laser pulse from the gain medium, $\phi_{i n}(\tau)$ and $\phi_{\text {out }}(\tau)$ stand for the phase of the input and output laser pulse, and $G(\tau)$ is the total gain experienced by the laser after passing through the gain medium, as defined by

$$
G(\tau)=\int_{0}^{L} g_{p}(z, \tau) d z
$$

Equation (8) can also be solved, as shown by

$$
\begin{aligned}
& P_{p}(L, \tau+\Delta \tau) \\
& \quad=P_{p}(\tau+\Delta \tau) \exp \left[-\int_{0}^{L} \alpha_{p}(z, \tau) z d z\right]
\end{aligned}
$$

where $P_{p}(\tau+\Delta \tau)=P_{p}(0, \tau+\Delta \tau)$ is the input pump power and $P_{p}(L, \tau+\Delta \tau)$ is the output pump power. By integrating (9) over $z$ from 0 to $L$, using (8), (13), (15), (17), (19), and (20), we obtain

$$
\begin{aligned}
\frac{d G(\tau)}{d \tau}= & \frac{P_{p}(\tau+\Delta \tau)}{\gamma E_{p \mathrm{sat}}} \\
& \cdot\left\{1-\exp \left[\gamma G(\tau)-b\left(N_{p 0}-N_{0}\right) L\right]\right\} \\
& -\frac{P_{\text {in }}(\tau)}{E_{\mathrm{sat}}}\{\exp [G(\tau)]-1\}
\end{aligned}
$$

By solving this differential equation, we may have the output pulse information from (17) and (18).

We find from (18) that the instantaneous frequency sweep $\Delta \omega$ of the laser pulse after passing through the gain medium is related to $G(\tau)$ by

$$
\Delta \omega_{\text {out }}=-\frac{d \phi_{\text {out }}(\tau)}{d \tau}=\Delta \omega_{\text {in }}+\frac{1}{2} \alpha \frac{d G(\tau)}{d \tau} .
$$

If the input laser pulse is nonchirped ( $\left.\Delta \omega_{\text {in }}=0\right), \Delta \omega_{\text {out }}$ may be expressed by

$$
\begin{aligned}
\Delta \omega_{\text {out }}= & \frac{\alpha P_{p}(\tau+\Delta \tau)}{2 \gamma E_{p \mathrm{sat}}} \\
& \cdot\left\{1-\exp \left[\gamma G(\tau)-b\left(N_{p 0}-N_{0}\right) L\right]\right\} \\
& -\frac{\alpha P_{i n}(\tau)}{2 E_{\mathrm{sat}}}\{\exp [G(\tau)]-1\} .
\end{aligned}
$$

Before going through the detailed numerical evaluation, we consider two extreme cases that have analytical solutions.

1) The first case is for no gain saturation, but including the pump bleaching effect. In this case, $E_{i n} \ll E_{s a t}$, where

$$
E_{i n}=\int_{-\infty}^{+\infty} P_{i n}(\tau) d \tau
$$

is the input laser pulse energy, we may thus ignore the second term on the right-hand side of (21) and (23). From (21), the gain $G(\tau)$ can be re-expressed by

$$
\begin{aligned}
G(\tau)= & a\left(N_{p 0}-N_{0}\right) L-\frac{1}{\gamma} \ln \left\{1+\left(e^{b N_{p 0} L}-1\right)\right. \\
& \left.\cdot \exp \left[-\frac{E_{p}(\tau+\Delta \tau)}{E_{p \mathrm{sat}}}\right]\right\}
\end{aligned}
$$

where

$$
E_{p}(\tau+\Delta \tau)=\int_{-\infty}^{\tau} P_{p}(\tau+\Delta \tau) d \tau .
$$

From (23) we find that the frequency sweep of the output pulse will follow the pump pulse shape $P_{p}(\tau+\Delta \tau)$ and be modified by the gain $G(\tau)$ as shown by

$$
\Delta \omega_{\text {out }}=R(\tau+\Delta \tau) \frac{P_{p}(\tau+\Delta \tau)}{\gamma E_{p \mathrm{sat}}}
$$

where

$$
\begin{aligned}
R(\tau+\Delta \tau) & =\frac{1}{2} \alpha \frac{\left(e^{b N_{p 00} L}-1\right) \exp \left[\frac{-E_{p}(\tau+\Delta \tau)}{E_{p \mathrm{sat}}}\right]}{1+\left(e^{b N_{p 0} L}-1\right) \exp \left[\frac{-E_{p}(\tau+\Delta \tau)}{E_{p \mathrm{sat}}}\right]} .
\end{aligned}
$$

Fig. 2(a) shows the instantaneous frequency sweep $\Delta \omega_{\text {out }}$ of a weak laser pulse passing through the gain medium under the average pump power of $150 \mathrm{~mW}\left(E_{p}=\right.$ $1.875 \mathrm{~nJ}$ ), which is a typical pump level for the GaAs mode-locked VCSEL. For comparison, we also show the cases for the average pump power of $1.5 \mathrm{~W}$ and $15 \mathrm{~mW}$. The pump pulse is shown by the dashed line. All other data needed for the calculation can be found in Table I. The laser pulse can be positioned anywhere depending on $\Delta \tau$, because the laser pulse is so weak that the laser chirp 


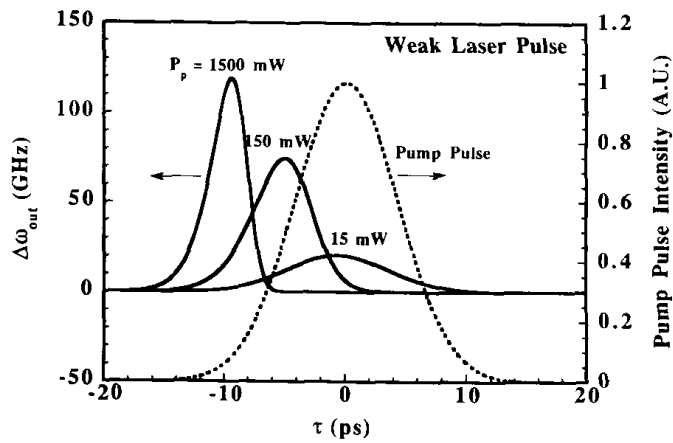

(a)

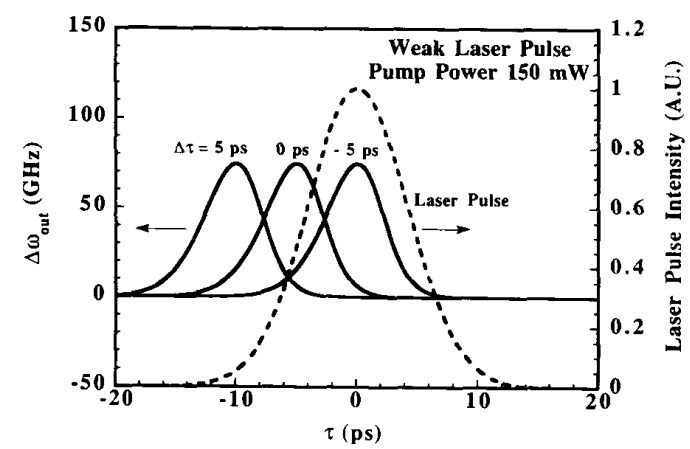

(b)

Fig. 2. (a) The instantaneous frequency sweep $\Delta \omega_{\text {sut }}$ of a weak laser pulse relative to the position of the pump pulse after a single pass through a 120 GaAs-AlGaAs multiple quantum-well (MQW's) sample at three different pump power $1.5 \mathrm{~W}, 150 \mathrm{~mW}$, and $15 \mathrm{~mW}$. The pump pulse of the $10 \mathrm{ps}$ is shown by the dashed line. The laser pulse can be positioned anywhere

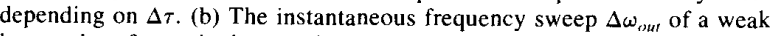
laser pulse after a single pass through a $120 \mathrm{GaAs}-\mathrm{AlGaAs}$ multiple quantum-well (MQW's) sample for three different position relative to the pump pulse $\Delta \tau$. The pump power is $150 \mathrm{~mW}$ and its pulsewidth is $10 \mathrm{ps}$. The laser pulse is shown by the dashed line.

TABLE I

\begin{tabular}{lc}
\hline Laser center wavelength $\lambda_{0}(\mu \mathrm{m})$ & 0.86 \\
Pump center wavelength $\lambda_{p}(\mu \mathrm{m})$ & 0.8 \\
Effective length of gain medium $L(\mu \mathrm{m})$ & 1.8 \\
Diameter of active medium $d(\mu \mathrm{m})$ & 19 \\
Differential gain at $\omega_{0} a\left(\mathrm{~cm}^{2}\right)$ & $5.27 \times 10^{-16}$ \\
Differential gain at $\omega_{p} b\left(\mathrm{~cm}^{2}\right)$ & $3.57 \times 10^{-15}$ \\
Differential gain ratio $\gamma$ & 6.77 \\
Transparent carrier density at $\omega_{0} N_{0}\left(\mathrm{~cm}^{-3}\right)$ & $6.97 \times 10^{17}$ \\
Transparent carrier density at $\omega_{p} N_{p 0}\left(\mathrm{~cm}^{-3}\right)$ & $1.98 \times 10^{18}$ \\
Carrier lifetime $\tau_{c}(\mathrm{~ns})$ & 3 \\
Intraband relaxation time $T_{2}(\mathrm{ps})$ & 0.1 \\
Linewidth enhancement factor $\alpha$ & 5 \\
Saturation energy at $\omega_{0} E_{\text {sat }}(\mathrm{nJ})$ & 1.24 \\
Saturation energy at $\omega_{p} E_{p s a t}(\mathrm{~nJ})$ & 0.20 \\
Pump pulsewidth $\tau_{p 0}(\mathrm{ps})$ & 10 \\
\hline
\end{tabular}

is totally determined by the pump pulse magnitude and the relative position between the pump pulse and the laser pulse $\Delta \tau$.

When the pump pulse energy $E_{p}$ is comparable to or smaller than the pump saturation energy $E_{p \mathrm{sat}}=0.2 \mathrm{~nJ}$, the laser frequency sweep $\Delta \omega_{\text {out }}$ will follow the pump pulse shape $P_{p}(\tau+\Delta \tau)$ as in the case of pump power of
$15 \mathrm{~mW}$. Depending on the laser pulse position relative to the pump pulse $\Delta \tau$, the laser will acquire either upchirp or downchirp. If the laser pulsewidth is smaller than the pump pulsewidth, the laser chirp can be linear for certain $\Delta \tau$.

When $E_{p}$ is larger than $E_{p \text { sat }}$ as in the cases of pump power of $150 \mathrm{~mW}$ and $1.5 \mathrm{~W}, \Delta \omega_{\text {out }}$ will rise at the leading edge of the pump pulse. Because of absorption bleaching, $\Delta \omega_{\text {out }}$ will immediately fall so that most parts of the laser pulse will acquire little frequency sweep if $\Delta \tau$ $>0$. Fig. 2(b) shows the instantaneous frequency sweep $\Delta \omega_{\text {out }}$ of the laser pulse for a pump power of $150 \mathrm{~mW}$ for various values of $\Delta \tau$. The laser pulse acquires significant chirp from the pump pulse only for the case of the laser pulse arriving in the gain region earlier than the pump pulse $(\Delta \tau<0)$. In a stablely operating synchronously pumped laser, the laser pulses usually arrive in the gain region later than the pump pulse $(\Delta \tau>0)$ [10].

2) The second case is for the absence of the pump, but with an initial gain $G_{0}$. We may obtain

$$
G(\tau)=G_{0} \exp \left[-\frac{E(\tau)}{E_{\mathrm{sat}}}\right]
$$

by ignoring the first term in (21) and assuming $G_{0} \ll<$, where

$$
E(\tau)=\int_{-\infty}^{\tau} P_{\text {in }}(\tau) d \tau
$$

The frequency sweep of the output laser pulse $\Delta \omega_{\text {out }}$ will follow the input laser pulse profile and be modified by the gain $G(\tau)$ as shown by

$$
\Delta \omega_{\text {out }}=-\frac{1}{2} \alpha G_{0} \exp \left[-\frac{E(\tau)}{E_{\text {sat }}}\right] \frac{P_{\text {in }}(\tau)}{E_{\text {sat }}}
$$

This case is essentially similar to that discussed in [9]. Fig. 3(a) shows the instantaneous frequency sweep $\Delta \omega_{\text {our }}$ of the laser pulse for various input pulse energies using the parameters given in Table I with $G_{0}=a\left(N_{p 0}-N_{0}\right) L$, the initial gain at which the gain medium is transparent to the pump pulse. When $E_{\text {in }} \ll E_{\text {sat }}$, $\Delta \omega_{\text {out }}$ shifts towards the negative following the laser pulse shape, so the chirp will be nonlinear over the central portion of the laser pulse. When $E_{\text {in }}>E_{\text {sat }}$, however, the saturation of the gain will alter the laser chirp. At the leading edge, the frequency sweep $\Delta \omega_{\text {our }}$ decreases following the rise of the laser pulse. The gain will then be saturated during the rise of the laser pulse so that $\Delta \omega_{\text {out }}$ will return immediately. Depending on the pulse energy level, the chirp acquired may be quite linear over the main portion of the laser pulse as shown by the curve for $E_{i n}=5 \mathrm{~nJ}$.

A saturable absorber case worth mentioning is when $G_{0}$ $=-a N_{0} L$ as shown in Fig. 3(b). When $E_{\text {in }} \ll E_{\text {sat }}, \Delta \omega_{\text {out }}$ simply follows the laser pulse shape. When $E_{\text {in }}>E_{\text {sat }}$, the saturation of the absorption will alter the laser chirp similar to the case of Fig. 3(a). At the leading edge, the frequency sweep $\Delta \omega_{\text {out }}$ increases following the rise of the laser pulse. The absorption will then be saturated during 


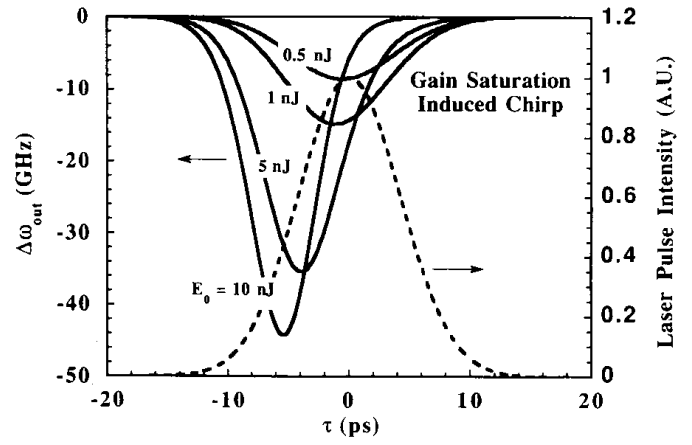

(a)

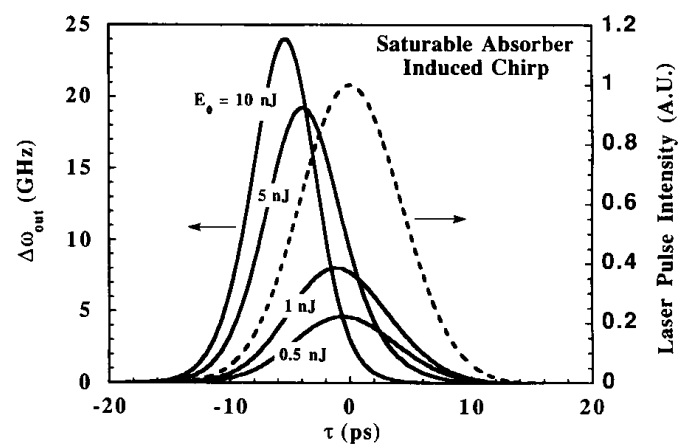

(b)

Fig. 3. The instantaneous frequency sweep $\Delta \omega_{\text {mu }}$ of a laser pulse with several different pulse energies after a single pass through a $120 \mathrm{GaAs}$ AIGaAs multiple quantum-well (MQW) sample without the pump, but with an initial gain (a) $G_{0}=a\left(N_{\rho 0}-N_{0}\right) L$, and (b) $G_{0}=-a N_{0} L$.

the rise of the laser pulse so that $\Delta \omega_{\text {out }}$ will fall immediately. Depending on the pulse energy level, the chirp acquired may also be quite linear over the main portion of the laser pulse as shown by the curve for $E_{\text {in }}=5 \mathrm{~nJ}$. The sign of chirp acquired by the laser pulse from the saturable absorber is the opposite to that acquired from the gain.

Concluding the discussions of (a) and (b), for a pump power of $150 \mathrm{~mW}$ and laser pulse energy of $5 \mathrm{~nJ}$, the pulsed optical pumping will cause a downchirp to the laser pulse because of the absorption bleaching, while the gain saturation will cause an unchirp to the laser pulse. To quantitatively evaluate the laser pulse chirp for the actual case of the mode-locked VCSEL, both effects should be taken into account together. We thus have to first obtain $G(\tau)$ numerically from (21) with both of the two right side terms involved, and then calculate the frequency sweep $\Delta \omega_{\text {out }}$ from (23). Under certain circumstances, either linear downchirp or linear upchirp could be obtained, which is important for laser pulse compression.

Fig. 4(a) and (b) shows the instantaneous frequency sweep $\Delta \omega_{\text {out }}$ of a $15 \mathrm{ps}$ pulse with a pulse energy of 10 and $5 \mathrm{~nJ}$, respectively, after passing through a sample with $120 \mathrm{GaAs}-\mathrm{AlGaAs}$ multiple quantum wells (MQW's). The pulse energy of $10 \mathrm{~nJ}$ corresponds to $24 \mathrm{~mW}$ average output power if the coupling mirror has a $97 \%$ reflectivity in the mode-locked VCSEL [1]. The typical pump power is $150 \mathrm{~mW}$ with 10 ps pulsewidth and $80 \mathrm{MHz}$ repetition

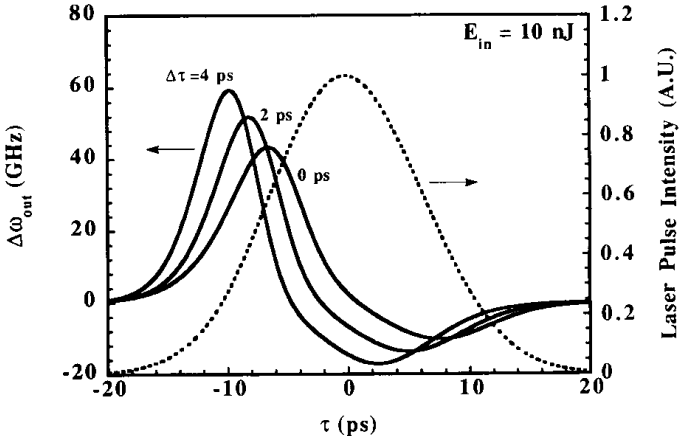

(a)

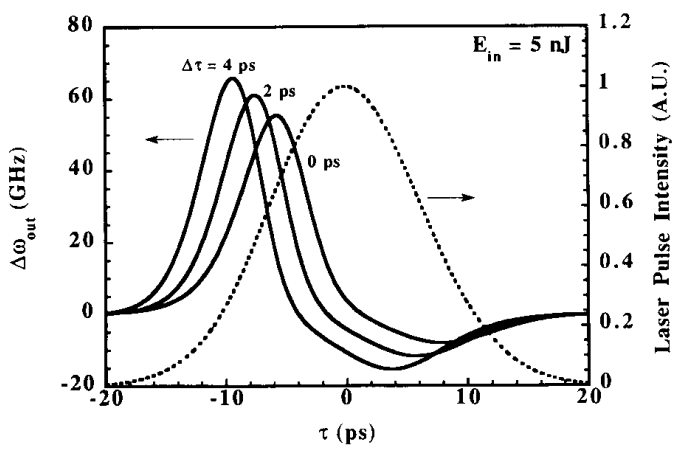

(b)

Fig. 4. The instantaneous frequency sweep $\Delta \omega_{\text {out }}$ of a $15 \mathrm{ps}$ pulse with a pulse energy of (a) $10 \mathrm{~nJ}$ and (b) $5 \mathrm{~nJ}$ after a single pass through a 120 GaAs-AlGaAs MQW sample with $\Delta \tau$ as a varying parameter. The pump pulsewidth is $10 \mathrm{ps}$ and pump power is $150 \mathrm{~mW}$.

rate. The rest of the parameters can be found in Table I. It is found that the central portion of the pulses is linearly downchirped when $\Delta \tau$ is small. If $\Delta \tau$ becomes larger, the frequency sweep increases but the chirp linearity decreases. For the same $\Delta \tau$, larger input pulse energy will create more linear chirp because of increased gain saturation.

The sign of the chirp on a single pass through the laser has a strong dependence on the laser pulsewidth if the pump pulsewidth is fixed. Fig. 5(a) and (b) shows the instantaneous frequency sweep $\Delta \omega_{\text {out }}$ of a $5 \mathrm{~nJ}$ laser pulse with different pulsewidth under $\Delta \tau=0$ and $2 \mathrm{ps}$, respectively. The pump pulsewidth is $10 \mathrm{ps}$ and pump power is $150 \mathrm{~mW}$. When $\Delta \tau=0$, the laser pulse chirp at its peak is negative if its pulsewidth is longer than 8 ps and becomes positive if its pulsewidth is shorter. When $\Delta \tau=2$ ps, there is a similar tendency, but the chirp will flip its sign for a laser pulsewidth of about 10 ps. Fig. 6 shows the instantaneous frequency sweep $\Delta \omega_{\text {out }}$ and the chirp $C$ at the pulse peak varying with the laser pulsewidth. The laser pulse energy is $5 \mathrm{~nJ}$ and $\Delta \tau=0$. The chirp $C$ is defined by

$$
C=-\frac{d^{2} \phi_{o u t}(\tau)}{d \tau^{2}}
$$

When the laser pulse is downchirped $(C<0)$, the frequency sweep at the pulse peak $\Delta \omega_{\text {out }}>0$. When the laser 


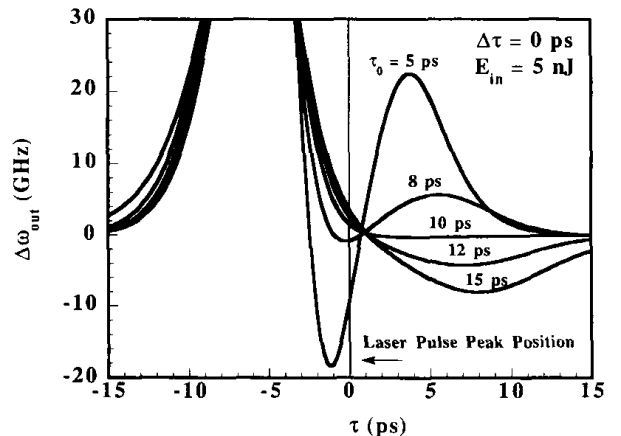

(a)

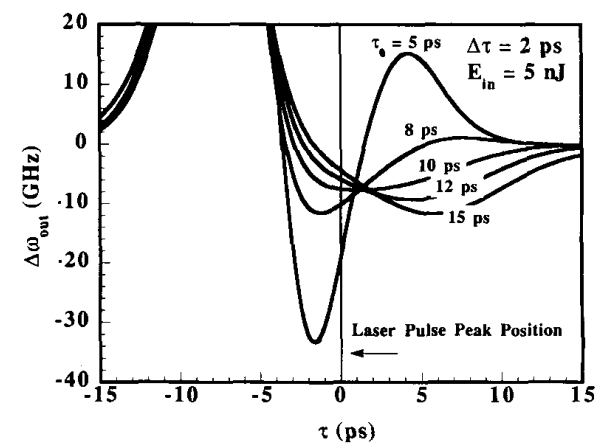

(b)

Fig. 5. The instantaneous frequency sweep $\Delta \omega$ of a $5 \mathrm{~nJ}$ laser pulse with its pulsewidth $\tau_{0}$ as a varying parameter after a single pass through a 120 GaAs-AIGaAs MQW sample under (a) $\Delta \tau=0$ and (b) $\Delta \tau=2$. The pump pulsewidth is $10 \mathrm{ps}$ and pump power is $150 \mathrm{~mW}$. The laser pulse peak position is shown by the vertical line in the center.

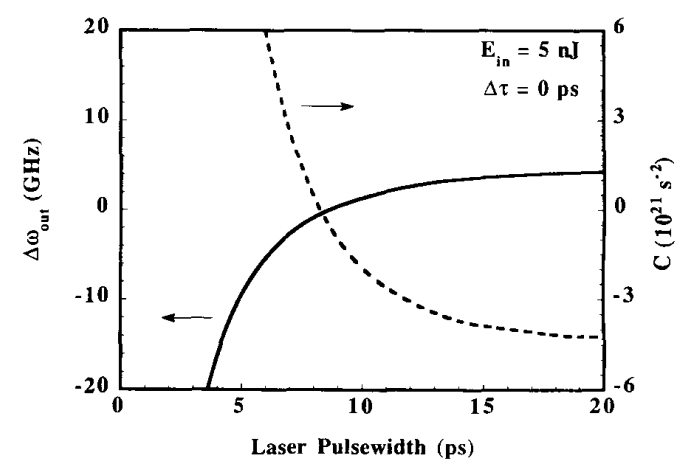

Fig. 6. The instantaneous frequency sweep $\Delta \omega_{o u}$ of a pulse and its chirp $C$ at the pulse peak after the single pass through a $120 \mathrm{GaAs}-\mathrm{AlGaAs} \mathrm{MQW}$ sample varying with the laser pulsewidth. The laser pulse energy is $5 \mathrm{~nJ}$ and $\Delta \tau=0$. The pump pulsewidth is $10 \mathrm{ps}$ and pump power is $150 \mathrm{~mW}$.

pulse is up-chirped $(C>0)$, the frequency sweep at the pulse peak $\Delta \omega_{\text {out }}<0$. Similar chirp behavior relating to the laser pulsewidth can be found for the wider pump pulsewidth.

To see how much linear group velocity dispersion (GVD) is needed to compensate for the SPM induced chirp because of the gain saturation and the pulsed pumping, we examine the phase of the output pulse obtained from (17), (18), and (21) in the frequency domain. The phase function $\Phi(\omega)$ in the frequency domain can be defined by $\Phi(\omega)=\beta(\omega) L$, where $\beta(\omega)$ is the wave propagation con- stant. We may expand $\Phi(\omega)$ in the Taylor series:

$$
\begin{aligned}
\Phi(\omega)= & \Phi\left(\omega_{0}\right)+\frac{d \Phi\left(\omega_{0}\right)}{d \omega}\left(\omega-\omega_{0}\right) \\
& +\frac{1}{2} \frac{d^{2} \Phi\left(\omega_{0}\right)}{d \omega^{2}}\left(\omega-\omega_{0}\right)^{2} \\
& +\frac{1}{6} \frac{d^{3} \Phi\left(\omega_{0}\right)}{d \omega^{3}}\left(\omega-\omega_{0}\right)^{3}+O\left(\omega-\omega_{0}\right)^{4} .
\end{aligned}
$$

The first derivative of the pulse phase with regard to the frequency $d \Phi\left(\omega_{0}\right) / d \omega$ is defined as the group delay. The net effect of this group delay is to create a local time shift $\Delta \tau$ for the whole pulse in the time domain. It can be compensated by varying the laser cavity length. What we are interested in is the second derivative of the pulse phase $d^{2} \Phi\left(\omega_{0}\right) / d \omega^{2}$, which is related to the dispersion parameter $\beta_{2}$ by $\beta_{2}=d^{2} \Phi\left(\omega_{0}\right) / d \omega^{2} / L$. Fig. 7(a) shows the variation of $d^{2} \Phi\left(\omega_{0}\right) / d \omega^{2}$ with $\Delta \tau$ for the laser pulse energy of $5 \mathrm{~nJ}$ and laser pulsewidth of $15 \mathrm{ps}$. Also shown in the figure is the gain $G(L)$ experienced by the laser pulse after it passes through the gain medium. When $d^{2} \Phi\left(\omega_{0}\right) / d \omega^{2}>0$, the pulse is upchirped, while the pulse is downchirped when $d^{2} \Phi\left(\omega_{0}\right) / d \omega^{2}<0$. For the laser, certain cavity gain is needed to overcome the cavity loss. For example, if the round-trip cavity loss is $5 \%$, a roundtrip gain of 1.05 will be needed to sustain the laser operation. Because of this extra gain requirement, the allowance of $\Delta \tau$ will be limited. For the example we have illustrated, the laser will be able to operate at certain range of $\Delta \tau>0$ so that $G(L)>1.05$. The laser pulse in this region will be downchirped with $d^{2} \Phi\left(\omega_{0}\right) / d \omega^{2}$ between $-9 \times 10^{-24} \sim-5 \times 10^{-24} \mathrm{~s}^{2}$. This value is four orders of magnitude as large as the GVD caused by the intracavity material dispersion or DBR structure of the semiconductor mirror and the dielectric mirrors.

We compare the magnitude of $d^{2} \Phi\left(\omega_{0}\right) / d \omega^{2}$ induced by the gain saturation and the pulsed optical pumping with that generated by a grating-pair. The dispersion from the grating-pair can be expressed by [11]

$$
\frac{1}{2} \frac{d^{2} \Phi\left(\omega_{0}\right)}{d \omega^{2}}=-\frac{4 \pi^{2} c b_{0}}{\omega_{0}^{3} a_{c}^{2} \cos ^{2} \vartheta_{r 0}}
$$

where $c$ is the speed of light, $b_{0}$ is the center to center distance between the gratings, $a_{c}$ is the grating constant (line spacing), and the diffraction angle $\theta_{r 0}$ is related to the incident angle $\theta_{i}$ by

$$
\sin \vartheta_{r 0}=\frac{2 \pi c}{\omega a}-\sin \vartheta_{i} .
$$

Both $\theta_{r 0}$ and $\theta_{i}$ are assumed on the same side of the grating normal. If assuming that the grating has 1200 lines $/ \mathrm{mm}, \lambda$ is $0.88 \mu \mathrm{m}$, and $\cos \theta_{r 0} \sim 1, d^{2} \Phi\left(\omega_{0}\right) / d \omega^{2}$ $\sim-5 \times 10^{-24} \mathrm{~s}^{2}$ will be equivalent to a $b_{0}$ of $\sim 1 \mathrm{~m}$. Since the dispersion obtainable from a prism-pair is much smaller than that from a grating-pair, such a large equivalent intracavity dispersion would be difficult to be compensated by the usual intracavity prism-pair dispersion compensation method [12] widely adopted in the colliding pulse mode locking (CPM) [13] and Ti : sapphire laser [14] systems, while the high loss of grating-pair [11], [15] 


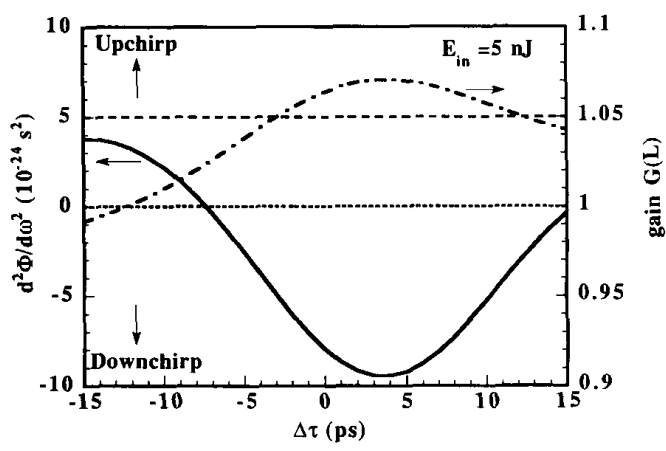

(a)

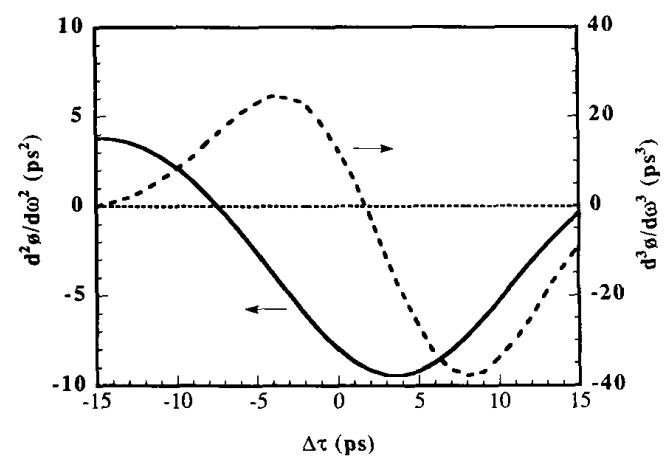

(b)

Fig. 7. (a) $d^{2} \Phi\left(\omega_{0}\right) / d \omega^{2}$ varying with $\Delta \tau$ after the laser pulse passing through a $120 \mathrm{GaAs}-\mathrm{AlGaAs} \mathrm{MQW}$ sample (solid line). Also shown in the figure are the gain $G(L)$ experienced by the laser pulse after its pass through the sample (dash-dotted line). The laser pulse energy is $5 \mathrm{~nJ}$ and the pulsewidth is $15 \mathrm{ps}$. The pump pulsewidth is $10 \mathrm{ps}$ and pump power is 150 $\mathrm{mW}$. The short dashed line specifies the gain level of 1 and the chirp level of 0 . The long dashed line specifies the gain level of 1.05 . (b) The variation of $d^{3} \Phi / d \omega^{3}$ with $\Delta \tau$ for the laser pulse energy of $5 \mathrm{~nJ}$ and pulsewidth of $15 \mathrm{ps}$ (dashed line). The pump power is $150 \mathrm{~mW}$ and the pump pulsewidth is $10 \mathrm{ps}$. The correspondent $d^{2} \Phi\left(\omega_{0}\right) / d \omega^{2}$ (solid line) is shown for comparison.

compressors prevents them from being used within the VCSEL cavity.

The third-order derivative of phase to the frequency $d^{3} \Phi\left(\omega_{0}\right) / d \omega^{3}$ reveals the nonlinearity of the chirp. Fig. 7(b) shows the variation of $d^{3} \Phi\left(\omega_{0}\right) / d \omega^{3}$ with $\Delta \tau$ for the laser pulse energy of $5 \mathrm{~nJ}$ and pulsewidth of $15 \mathrm{ps}$. The pump power is $150 \mathrm{~mW}$ and the pump pulsewidth is 10 ps. For comparison, $d^{2} \Phi\left(\omega_{0}\right) / d \omega^{2}$ is also shown in the same figure. Notice that we can find a position $\Delta \tau=1.7$ ps, so that $d^{3} \Phi\left(\omega_{0}\right) / d \omega^{3}=0$. Physically it means that at this position the chirp around the peak of the laser pulse has the best linearity. The consequence is that the chirp of the output pulse can thus be ultimately compensated by a grating-pair which has only the linear dispersion.

\section{Gain Dispersion}

The chirp caused by the SPM will broaden the spectrum of the pulse so that it will eventually reach such a width that the finite gain shape of the gain material or other magnitude filtering elements may limit its further broadening. In this section, we will discuss the effect of the phase dispersion caused by the finite gain shape. The gain saturation and the linewidth enhancement factor $\alpha$ will be ignored during the discussion since their role related to the SPM has been taken into account in the last section. This procedure is similar to the split-step Fourier method [8] which has been used in the analysis of light transmitting in the optical fiber.

We assume that the gain shape of the semiconductor materials is Lorenzian so that the comple $_{A}$ power gain coefficient can be written as [16]

$$
\tilde{g}\left(\omega-\omega_{0}\right)=\frac{g_{p}}{1-i T_{2}\left(\omega-\omega_{0}\right)}
$$

where $g_{p}$ is the peak power gain coefficient, $\omega_{0}$ is the peak frequency, and $T_{2}$ is a bandwidth parameter. The exact gain shape of the semiconductor materials is somewhat different in that the gain shape is closer to the Gaussian shape on the higher frequency side [17]. The difference will, however, not affect the discussion here.

In the frequency domain, (6) should be written as

$$
\frac{\partial \tilde{A}(z, \omega)}{\partial z}=\frac{1}{2} \tilde{g}\left(\omega-\omega_{0}\right) \tilde{A}(z, \omega)
$$

where $\tilde{A}(\omega)$ is related to $A(z, \tau)$ by the inverse Fourier transform

$$
A(z, \tau)=\int_{-\infty}^{\infty} \tilde{A}(z, \omega) e^{-i\left(\omega-\omega_{0}\right) \tau} d \omega .
$$

After a single pass through the gain medium of length $L$, the electric field $\tilde{A}(L, \omega)$ can be solved in terms of the input $\tilde{A}(0, \omega)$ from (37), as shown by

$$
\tilde{A}(L, \omega)=\tilde{A}(\omega) \exp [i \Phi(\omega)]
$$

where the real amplitude

$$
\tilde{A}(\omega)=\tilde{A}(0, \omega) \exp \left(\frac{\frac{1}{2} g_{p} L}{1+T_{2}^{2}\left(\omega-\omega_{0}\right)^{2}}\right)
$$

and the phase

$$
\Phi(\omega)=\frac{\frac{1}{2} g_{p} L T_{2}\left(\omega-\omega_{0}\right)}{1+T_{2}^{2}\left(\omega-\omega_{0}\right)^{2}} .
$$

The peak gain $g_{p}$ can be obtained from (25) as

$$
g_{p}=a\left(N_{p 0}-N_{0}\right)
$$

by assuming $E_{p} \gg E_{p \text { sat }}$, which is true for the modelocked VCSEL. From (41), it is found that the maximum value of the second derivative of the phase, $\Phi_{\max }^{\prime \prime}(\omega)$, can be expressed by

$$
\Phi_{\max }^{\prime \prime}=0.73 g_{p} L T_{2}^{2} .
$$

Using the data in Table $I$, we find that $\Phi_{\max }^{\prime \prime}(\omega)$ is $\sim 1 \times 10^{-27} \mathrm{~s}^{2}$. This is three orders of magnitude smaller that the $\Phi^{\prime \prime}(\omega)$ caused by the gain saturation and the pulsed pumping. In other words, the phase dispersion caused by the finite gain shape will have little effect on the formation of the laser pulse chirp in a mode-locked VCSEL. 


\section{Discussion of Laser Pulse Evolution}

Laser pulse evolution in the laser cavity involves pulse shortening mechanisms and pulse broadening mechanisms. In the frequency domain, it is a balance between spectral broadening and spectral narrowing. In a synchronously pumped laser system, the leading edge of the laser pulse is narrowed by the rising of the gain, the trailing edge is confined by saturation of the gain. This gain modulation mechanism causes pulse shortening. In the frequency domain, pulse shortening is represented by spectral broadening. For the mode-locked VCSEL, strong SPM will cause extra spectral broadening, thus the timebandwidth product will be larger than the Fourier transform limit. With the shortening of the pulsewidth and the broadening of the spectrum, the dispersive elements in the laser cavity start to act on the laser pulse. These dispersive elements include material dispersion, phase dispersion of gain, and DBR structure related dispersion. The dispersion can be a pulse shortening factor or pulse broadening factor, depending on the relative sign between the SPM and the dispersion. The material dispersion factor is small, however, compared to the SPM effects as shown in the discussions in Section II and III. One of the dominant pulse broadening or spectral narrowing mechanisms is a spectral filter with a finite filter bandwidth within the laser cavity. This filter can be an intracavity birefringent filter, an etalon, the Fabry-Perot effect in an imperfect intracavity element, the finite gain bandwidth or something else related to the laser cavity design. Carrier transport because of the nonuniform carrier distribution within the gain medium will also play an important role in limiting the laser pulse shortening, which is to be addressed in detail somewhere else [18].

Let's look at what will happen if an initially unchirped pulse of $15 \mathrm{ps}$ is launched into the GaAs VCSEL. The pulse will become downchirped because of the SPM due to the gain saturation and the pulsed optical pumping (Fig. 6) after one round-trip traveling in the cavity. During the second round-trip, the downchirped input pulse becomes more downchirped at the output because of the SPM [Fig. $8(\mathrm{a})]$. This process will continue so that the laser pulse will be shortened and the laser spectrum will be broadened. Interestingly, the downchirp acquired by the laser pulse during each round-trip around the laser cavity tends to decrease after several passes because of the pulse shortening (Fig. 6). We examine two important cases of the equilibrium pulse formation processes.

In the first case, the filter bandwidth is not very wide, but the spectrum of the laser pulses has become as wide as that of the spectral width of a Fourier transform limited subpicosecond pulse. The actual pulsewidth is still more than 8 ps though since the pulse is strongly chirped. Further spectral broadening is suppressed by the filter effect within the cavity. On each additional pass through the gain medium, the laser pulse will acquire extra bandwidth because of the gain modulation and the SPM. The acquired extra bandwidth will be cut by the filter so that after a round-trip, the pulse spectrum remain unchanged. In the time domain, this will be the case that the pulse shorten-

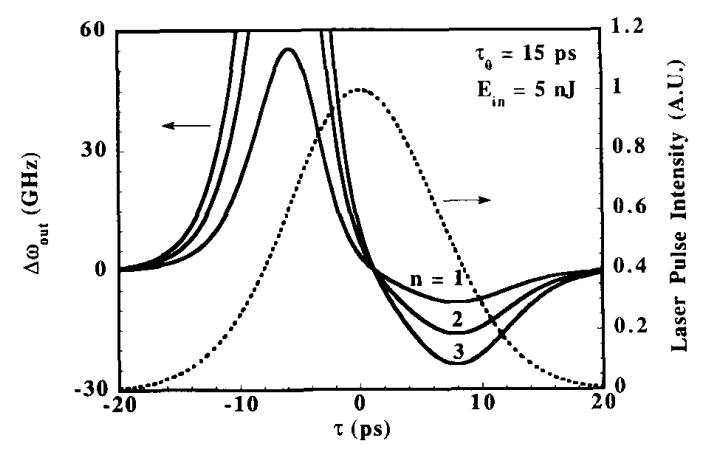

(a)

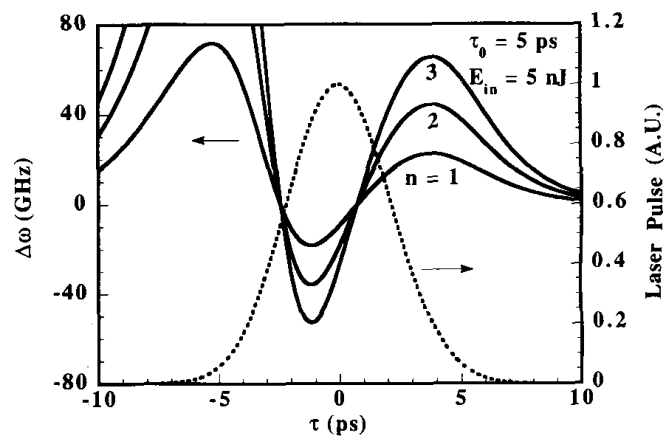

(b)

Fig. 8. The instantaneous frequency sweep $\Delta \omega_{\text {rut }}$ (solid lines) of a laser pulse after the first three passes through a $120 \mathrm{GaAs}-\mathrm{AlGaAs}$ MQW sample. The laser pulsewidth $\tau_{0}$ is (a) $15 \mathrm{ps}$ and (b) $5 \mathrm{ps}$, and laser pulse energy $E_{i n}$ is $5 \mathrm{~nJ}$ (short dashed line). The pump pulsewidth is $10 \mathrm{ps}$ and pump power is $150 \mathrm{~mW}$.

ing is balanced by the pulse broadening. The output pulse will be down-chirped. This is the case we have obtained experimentally [1].

In the second case, the filter bandwidth is very wide and the gain modulation induced pulse shortening is strong enough to obtain a pulsewidth shorter than $8 \mathrm{ps}$. The sign of the laser pulse chirp acquired during each additional pass through the gain medium will thus be positive as shown in Fig. 6. This will counter-act the down-chirp acquired by the laser pulse in the former trips so that the laser pulse will soon become up-chirped. As the laser pulse becomes more and more up-chirped [Fig. 8(b)], the spectral broadening caused by the gain modulation and the SPM due to gain saturation and pulsed optical pumping will be offset by the filter. The laser pulsewidth will thus be stabilized, and the laser pulse will be up-chirped. We are unable to reconstruct this case experimentally with the pump pulsewidth of $10 \mathrm{ps}$ or shorter. It can be done, however, with a pump pulsewidth of $100 \mathrm{ps}$. In this case, a laser pulse of shorter than 30 ps pulsewidth passing through the gain medium will acquire positive chirp, rather than the down chirp in the case of the pump pulsewidth of $10 \mathrm{ps}$. The laser pulse will thus have an upchirp when it is stabilized in the laser cavity. This is the case observed in the InGaAs-InP laser system [2], [3].

According to the above discussions, the laser pulse can be made unchirped with the insertion of matched filter in 
the cavity to limit extra bandwidth. This approach has been reported by Sun and Yamamoto [19], who have utilized the coupled-cavity characteristics which works like a filter in the cavity. Another possibility will be to utilize the downchirp characteristics of a saturable absorber [Fig. 3(b)]. This approach involves how to choose parameters of saturable absorbers and is only appropriate for the compensation of pulses with upchirp.

It should be pointed out that in some occasions the intracavity filter effect may not be the mechanism in limiting the laser pulse shortening, but the finite carrier transport time within the gain medium. Such a difference, however, does not affect the basic idea of the pulse formation discussion. To conclude the discussion of this section, it is worth mentioning that the laser chirp is linearly proportional to the linewidth enhancement factor $\alpha$ according to (18). The best way to decrease the laser chirp is to have a smaller $\alpha$.

\section{Discussions of Experimental Results}

It was reported that in a GaAs mode-locked VCSEL the pulse was downchirped [1]. A grating-pair with a telescope setup was used to generate positive dispersion to compensate for the chirp. Other reports revealed that pulses from InGaAs-InP mode-locked VCSEL's were upchirped [2], [3]. The variation of the chirp sign can be explained from Figs. 5 and 6. In the GaAs VCSEL, the laser pulse was always longer than $10 \mathrm{ps}$ while the pump laser pulsewidth was only $5 \mathrm{ps}$. Accordingly, the laser pulse must have been downchirped-the first case discussed in Section IV. In the InGaAs-InP VCSEL, the pump pulsewidth was over 150 ps [3] and the laser pulses varied from 5 to $40 \mathrm{ps}$, which were shorter than the pump pulsewidth, thus the laser pulses were upchirped-the second case discussed in Section IV. In the case of [2], although the laser pulse was about $7 \mathrm{ps,} \mathrm{longer} \mathrm{than} \mathrm{the}$ pump pulsewidth, it was actually the addition of several pulses very close to each other. The actual pulsewidth of the individual pulses was shorter than the pump pulse. Also, the pump came from the compression of a $120 \mathrm{ps}$ pulse by a fiber grating-pair compressor, so that the compressed pulse had a relatively long tail, which differed from the pump pulse shape of Gaussian we have assumed throughout this discussion. Different shapes of the pump pulse will make the laser pulse to change its chirp sign at different laser pulsewidth, but will not change the basic discussions we have made.

It has been found experimentally that the laser chirp is widely tunable by varying the laser cavity length. It is understandable from Figs. 4 and 7 that the variation of $\Delta \tau$ will change not only the magnitude of chirp, but also the linearity of the chirp. More importantly, the laser pulsewidth is critically dependent on the matching of the laser cavity length and the pump cavity length in a synchronously pumped laser system. The variation of the laser of the laser cavity length will vary the relative position between the laser and the pump pulses $\Delta \tau$, thus severely varying the laser pulsewidth and altering the pulse chirp as shown by Figs, 5 and 6 .

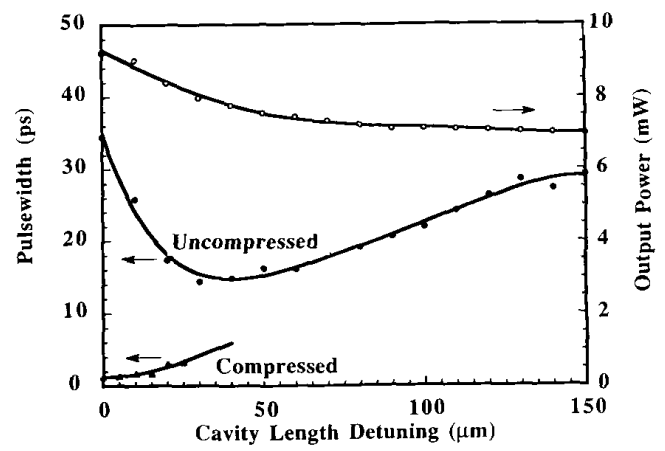

Fig. 9. The laser pulsewidth and its output power versus the cavity detuning in a mode-locked GaAs VCSEL. Also shown is the variation of the compressed pulsewidth with the cavity detuning. The solid lines are a guide to the eye.

Fig. 9 shows the laser pulsewidth and the output power as a function of the detuning of the laser cavity in a modelocked GaAs VCSEL. The pump pulsewidth is $10 \mathrm{ps}$ and the pump power is $150 \mathrm{~mW}$. Also shown in Fig. 9 is the compressed pulsewidth. Note that efficient pulse compression occurs only for a very narrow region close to the maximum output power and where the uncompressed pulsewidth is not at the minimum. A similar observation was found in the InGaAs-InP system [3] where the maximum compression occurred when the uncompressed pulsewidth was $30 \mathrm{ps,}$, rather then the shortest pulsewidth of 5 ps.

The phenomena are also explainable. We find in Fig. 7 (a) that the maximum net gain happens at $\Delta \tau \sim 3 \mathrm{ps}$ for the laser pulsewidth of $15 \mathrm{ps}$ and laser pulse energy of 5 $\mathrm{nJ}$, rather than the cavity length matching case of $\Delta \tau=0$ ps. At this position, the chirp of the laser pulse is more linear than the cases of larger $\Delta \tau$ [Fig. $7(\mathrm{~b})]$, thus the compression is more effective. To optimize the laser pulsewidth in a synchronously pumped laser system, however, the laser cavity should be detuned longer, rather to be the maximum gain position, so that the gain saturation can be fully used to suppress the trailing edge of the laser pulse [10]. For a larger $\Delta \tau$, the chirp linearity deteriorates, thus the compression becomes less efficient.

\section{Conclusion}

We have discussed the origin of the laser pulse chirp in mode-locked VCSEL's. Gain saturation and pulsed optical pumping are the dominant causes of the pulse chirping. The adjustment of the chirp value can be realized mainly through tuning the laser cavity length thus varying the laser pulsewidth. The sign of the laser chirp is closely related to the laser pulsewidth and the pump pulsewidth, as well as the relative importance of the gain saturation and the pulsed optical pumping. The magnitude of the chirp is so large tha the usual intracavity prism-pair chirp compensation becomes impractical. This problem is intrinsic to the synchronously pumped VCSEL system since larger pulse energy is always related to a larger variation of carrier density, producing larger chirp. External cavity chirp compensation seems efficient [1]-[3], but the com- 
pressed pulses often contain a large amount of energy in wings due to the difficulty in compensation for the nonlinear chirp over the part other than the central portion of the pulses. An extra intracavity filter will be a useful way to limit the spectral width so that the time bandwidth product of pulses can be made closer to the Fourier transform limit, but it will also limit the minimum pulsewidth to be achieved. Saturable absorbers will probably be a candidate for the intracavity chirp compensation if the laser is upchirped as in the case of InGaAs mode-locked VCSEL's. They will not be useful for the chirp compensation purpose if the laser is downchirped as in the case of GaAs mode-locked VCSEL.

\section{ACKNOWLEDGMENT}

The authors acknowledge the helpful discussions with Dr. M. Shimizu, D. I. Babic, and R. J. Helkey. Thanks are also due to R. P. Mirin for the sample growth.

\section{REFERENCES}

[1] W. B. Jiang, R. Mirin, and J. E. Bowers, "Mode-locked GaAs vertical cavity surface emitting lasers," Appl. Phys. Lett., vol. 60, pp. $677-679,1992$.

[2] W. B. Jiang, S. R. Friberg. H. Iwamura, and Y. Yamamoto, "High powers and subpicosecond pulses from an external-cavity surfaceemitting InGaAs/InP multiple quantum well laser," Appl. Phys Lett., vol. 58, pp. 807-809, 1991.

[3] W. H. Xiang, S. R. Friberg, K. Watanabe, S. Machida, W. B. Jiang. H. Iwamura, and Y. Yamamoto, "Femtosecond external-cavity surface-emitting InGaAs/InP multiple-quantum-well laser," Opt. Lett., vol. 16, pp. 1394-1396, 1991.

[4] W. H. Xiang. S. R. Friberg, K. Watanabe, S. Machida, Y, Sakai, H. Iwamura, and Y. Yamamoto, "Sub-100 femtosecond pulses from an external-cavity surface-emitting $\operatorname{lnGaAs} / \ln \mathrm{P}$ multiple quantum well laser with soliton-effect compression," Appl. Phys. Lett., vol. 59. pp. 2076-2078, 1991.

[5] S. de Silvestri, P. Laporta, and O. Svelto, "The role of cavity dispersion in CW mode-locked lasers," IEEE J. Quantum Electron., vol. QE-20, pp. 533-539. 1984 .

[6] P. Laporta and V. Magni, "Dispersive effects in the reflection of femtosecond optical pulses from broadband dielectric mirrors," Appl. Opt., vol. 24, pp. 2014-2020, 1985.

[7] A. N. Pikhtin and A. D. Yas'kov, "Dispersion of the refractive index of semiconductors with diamond and zinc-blende structures," Sov. Phys. Semicond., vol. 12, pp. 622-626, 1978.

[8] G. P. Agrawal. Nonlinear Fiber Optics. New York: Academic, 1989. ch. 1 and 2 .

[9] G. P. Agrawal and N. A. Olsson, "Self-phase modulation and spectral broadening of optical pulses in semiconductor laser amplifiers," IEEE J. Quantum Electron., vol. 25, pp. 2297-2306, 1989.

[10] D. M. Kim, J. Kuhl, R. Lambrich, and D. von der Linde, "Characteristics of picosecond pulses generated from synchronously pumped CW dye laser system," Opt. Commun., vol. 27, pp. 123-126, 1978.

[11] E. B. Treacy, "Optical pulse compression with diffraction gratings," IEEE J. Quantum Electron, vol. QE-5, pp. 454-458, 1969.

[12] R. L. Fork, O. E. Martinez, and J. P. Gordon, "Negative dispersion using pairs of prisms," Opt. Lett., vol. 9, pp. 150-152, 1984.

[13] W. H. Xiang, W. B. Jiang, and Y. Ishida, "Femtosecond pulses generated from non-colliding pulse mode-locked ring dye lasers." Opt. Commun., vol. 86, pp. 70-74, 1991.

[14] N. Sarukura, Y. Ishida, and H. Nakano, "Generation of 50-fsec pulses from a pulse-compressed, $c w$ passively mode-locked Ti : sapphire laser," Opt. Lett, vol. 16, pp. 153-155, 1991.

[15] O. E. Martinez, "3000 times grating compressor with positive group velocity dispersion: application to fiber compensation in $1.3-1.6 \mu \mathrm{m}$ region," IEEE J. Quantum Electron., vol. QE-23, pp. 59-64, 1987.

[16] A. E. Siegman, Lasers. Mill Valley, CA: Univer. Sci. Books, 1986, ch. 8 . [17] A. Yariv, Quanum Electronics. New York: Wiley, 1989, 3rd ed.,
ch. 21 .
[18] W. B. Jiang, M. Shimizu, R. P. Mirin, T. E. Reynolds, and J. E. Bowers, "Fentosecond periodic gain vertical-cavity lasers," IEEE Photon. Technol. Lett., vol. 5, pp. 23-25, 1993.

[19] D. C. Sun and Y. Yamamoto, "Passive stabilization of a synchronously pumped external-cavity surface-emitting InGaAs / InP multiple quantum well laser by a coherent photon-seeding technique," Appl. Phys. Lett., vol. 60, pp. 1286-1288, 1992.

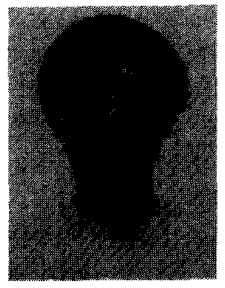

Wenbin Jiang received the B.S. degree in physics and the M.S. degree in optics from Fudan University, Shanghai, in 1985 and 1988 , respectively.

$\mathrm{He}$ is currently a Ph.D. candidate with the Department of Electrical and Computer Engineering. University of California, Santa Barbara. From 1988 to 1989 , he was with NTT Basic Research Labs, Tokyo, working on two-color soliton collisions for QND measurement, passive mode locking of color center lasers, and mode locking of surface-emitting semiconductor lasers. His primary research is on modelocked surface-emitting laser device fabrication and measurement.

Mr. Jiang is a student member of the Optical Society of America and the American Physical Society.

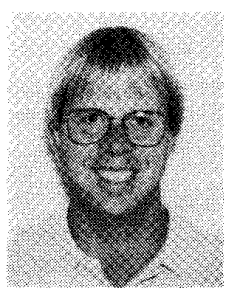

Dennis J. Derickson (M'87) was born in Westbrook, MN, in 1959. He received the B. S. degree in electrical engineering in 1981 from South $\mathrm{Da}$ kota State University, Brookings, and the M.S. degree in electrical engineering in 1982 from the University of Wisconsin, Madison. In 1992 he received the Ph.D. degree in electrical engineering from the University of California, Santa Barbara.

From 1983 to 1988 he was a member of the technical staff at Hewlett-Packard Co., Santa Rosa, CA, working on GaAs monolithic amplifiers, millimeter wave filters, and high-speed optical to electrical converters. His current research interests are in the area of optoelectronic device design and fabrication.

Dr. Derickson is a member of the Optical Society of America and the American Physical Society.

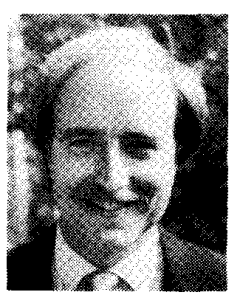

John E. Bowers (S'78-M'81-SM'85-F'93) received the M.S. and Ph.D. degrees in applied physics from Stanford University, Stanford, CA. His dissertation involved the design, fabrication, and demonstration of SAW signal processing devices.

He was a research associate at Stanford University conducting research on single-mode fiber optic devices, sensors, and signal processors. he was with Honeywell in 1979, where he developed an LPE system for the large area multilayer growth of $\mathrm{HgCdTe}$. From 1982 to 1987, he was with Bell Laboratories, Holmdel, $\mathrm{NJ}$, investigating quaternary lasers, modulators, photodetectors, and highspeed and subcarrier transmission systems. He was part of the teams that demonstrated the first $8 \mathrm{Gbit} / \mathrm{s}$ and $16 \mathrm{Gbit} / \mathrm{s}$ transmission systems. He is currently a professor at the University of California, Santa Barbara, and a member of the Optoelectronics Technology Center and the NSF Science and Technology Center on Quantized Electronic Structures. His research interests are primarily concerned with high frequency optoelectronic devices and the physics of femtosecond processes and quantum structures.

Dr. Bowers is a member of the American Physical Society, the Optical Society of America, and the International Society for Optical Engineering (SPIE). He is a recipient of Sigma Xi's Thomas F. Andrew's prize for undergraduate research and the National Science Foundation's Presidential Young Investigator Award. He is chair of the LEOS technical subcommittee on semiconductor lasers. 